\title{
Pseudosarcomatous Fibromyxoid Tumor of the Bladder and Prostate: A Case Report
}

\author{
Jason R. Lomboy ${ }^{1}$, Tejas Desai ${ }^{* 2}$ and J. Corey Allen ${ }^{3}$ \\ ${ }^{1}$ Brody School of Medicine at East Carolina University, Greenville, NC, USA \\ ${ }^{2}$ Department of Internal Medicine, Division of Nephrology and Hypertension, Brody School of Medicine at East \\ Carolina University, Greenville, North Carolina, USA \\ ${ }^{3}$ Eastern Urological Associates, P.A., Greenville, North Carolina, USA
}

\begin{abstract}
Pseudosarcomatous fibromyxoid tumor (PSFT) is a rare pseudotumor of the genitourinary (GU) system that typically presents with gross hematuria. These tumors are benign, locally aggressive tumors. Metastasis has never been reported. Pathology is significant for spindle cell proliferation without malignant nuclear features that are commonly seen in sarcomas. Herein, we report a case of an 8-centimeter PSFT found within the bladder of a 71-year-old gentleman with gross hematuria. We also review the literature on genitourinary pseudotumors, including postoperative spindle cell nodules (PSCN). Lastly we describe the importance of considering these types of tumors in the presence of a GU mass as early identification may preclude the patient from receiving unnecessary imaging and aggressive treatment measures.
\end{abstract}

Keywords: Inflammatory pseudotumor, pseudosarcoma, pseudosarcomatous fibromyxoid tumor, postoperative spindle cell nodule, nodular fasciitis, pseudosarcomatous myofibroblastic tumor, fibromyxoid pseudotumor, pseudo-malignant spindle cell proliferation, inflammatory myofibroblastic tumor.

\section{INTRODUCTION}

Pseudosarcomas of the genitourinary tract, first described in 1980 by Roth [1] as a reactive pseudosarcomatous response, are uncommon, benign lesions of the urinary tract. They are unique lesions in the manner in which they are easily mistaken for malignant sarcomas upon gross inspection. While they are known by many different names in the literature-including nodular fasciitis, pseudosarcomatous myofibroblastic tumor, fibromyxoid pseudotumor, pseudo-malignant spindle cell proliferation, inflammatory myofibroblastic tumor, and inflammatory pseudotumor [2]the most commonly referenced names are pseudosarcomatous fibromyxoid tumor (PSFT) and postoperative spindle cell nodule (PSCN). The two lesions are essentially identical histologically, and very little evidence in the literature has found reliable methods to separate the two lesions aside from history of surgical instrumentation of the urinary tract.

We present a case report in which a 71-year-old gentleman with no history of urologic surgery or instrumentation who presented with several episodes of gross hematuria and was found to have a PSFT larger than 8 centimeters emanating from the prostate and into the bladder.

\section{CASE REPORT}

A 71-year-old gentleman presented from an outside hospital with a 2 day history of gross hematuria and nocturia

*Address correspondence to this author at the Department of Internal Medicine, Division of Nephrology and Hypertension, Brody School of Medicine at East Carolina University, Greenville, North Carolina, USA; Tel: 252-744-1380; E-mail: tejas.p.desai@gmail.com up to eight times a night. The patient reported having gross hematuria intermittently for the past several years but denied any further work-up. The patient did not report other urinary symptoms, fever, or pain. The patient had a past medical history of hypertension, controlled with diet and exercise. Prior surgeries included a hemorroidectomy. Social history revealed patient was a prior smoker. Vital signs on admission were stable with blood pressure 150/91, temperature 36.9 degrees Fahrenheit, pulse 90 beats per minute, and respiratory rate 18 . Physical exam was relatively benign, with no signs of distress, focal tenderness, or anemia. Patient was unable to tolerate an adequate prostate examination.

A CT urogram on this admission showed marked prostatomegaly with a pedunculated mixed density mass with heterogeneous enhancement indistinguishable from the prostate gland extending into the lumen of the bladder. The intraluminal portion was measured to be $8.3 \times 6.8 \times 8.1 \mathrm{~cm}$. Representative images are present in Fig. (1). Following 3 days of hand irrigation and CBI, the patient's urine became clear, and he was discharged home with a 24 French 3-way catheter. A plan for a cystoscopy and transurethral resection of bladder tumor (TURBT) was scheduled 5 days later.

Cystoscopy revealed obstructing and friable lateral lobes as well as nodular lesions particularly in the left lateral lobe of the prostate. A very large mass occupied the majority of the bladder, which appeared to be originating from the bladder neck. There was an additional raised erythematous lesion on the right lateral bladder wall. Five separate biopsies were taken from the right lateral bladder wall, bladder mass, bladder neck, left lateral prostatic lobe, and distal prostatic 


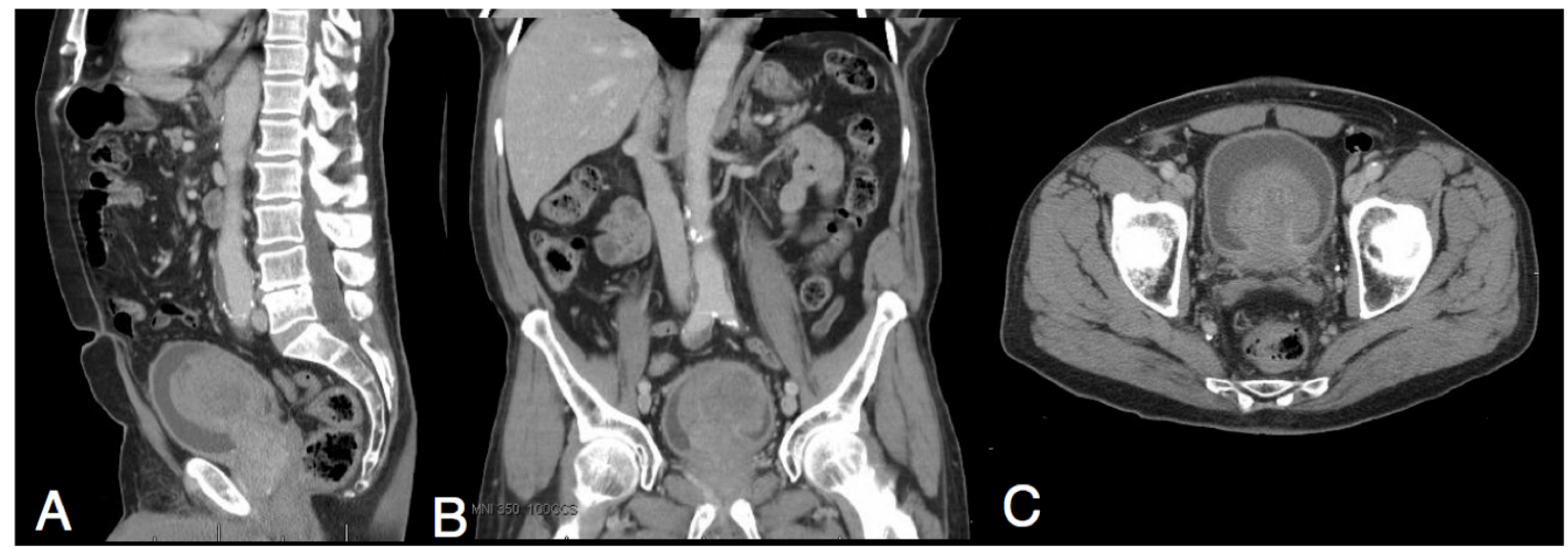

Fig. (1). Representative cuts from a computed tomography (CT) scan of patient's abdomen and pelvis showing an 8-centimeter mass from the prostate gland extending into the lumen of the bladder. (A) Sagittal view (B) Coronal view (C) Transverse view.

urethra. Pathology revealed pseudosarcomatous fibromyxoid tumor in the bladder mass, bladder neck, and left lateral prostatic lobe. Additionally focal urothelial dysplasia was identified from the bladder wall specimen. Immunostains with Ki67 and CK20 were performed with adequate controls. The Ki67 stain showed increased positive cells in the upper urothelium. After continuous bladder irrigation (CBI) with catheter traction, the patient's urine cleared and he was discharged home with a Foley catheter and a plan for suprapubic prostatectomy.

Following two Emergency Department visits for gross hematuria in the interim, the patient returned 17 days later for his operation. Following the suprapubic prostatectomy, pathology confirmed the diagnosis of pseudosarcomatous fibromyxoid tumor. The patient had an uneventful postoperative course and was discharged home. The patient followed up as an outpatient, his catheters were removed, and he has been voiding without any complaints.

\section{DISCUSSION}

Pseudosarcomas of the genitourinary tract are uncommon lesions whose pathogenesis is poorly understood. On gross inspection, they may resemble aggressive sarcomas. Histologically, these tumors are diagnosed by the presence of spindle and stellate cell proliferation without active mitoses in a background myxoid stroma [3]. With only sparse case reports existing in the literature, naming and classification have been inconsistent. The most commonly used terms include pseudosarcomatous fibromyxoid tumor (PSFT) and postoperative spindle cell nodules (PSCN). These lesions together are not only histologically identical, but they also present similarly, have a similar prognosis, and are cured typically by surgical excision alone.

Pathologists and urologists have struggled with the classification of lesions with pseudosarcomatous histology. With a multitude of names for these lesions existing in the literature, the focus has been on distinguishing spontaneous pseudosarcomatous fibromyxoid tumors (PSFT) from postoperative spindle cell nodules (PSCN). Multiple case studies have reported on PSFT of the bladder and prostate in addition to sparse reports of these lesions located in other areas of the urogenital system, including the ureter [4], vagina [5], and urethra [6]. Postoperative spindle cell nodules (PSCN), first recognized in 1984 by Proppe et al. [7], are found only in patients with a history of urologic surgery or prior urinary tract instrumentation. One study involving 38 instances of spindle cell tumors concluded that PSCN differ from spontaneous inflammatory pseudotumors such as PSFT in that PSCN involved older patients, smaller average tumor size, the presence of eosinophils, and a higher mitotic rate [8]. Another study also agreed with differences in age at presentation [9]. Additionally, this study found that PSFT were most often encountered after work-up of painless, gross hematuria, while PSCN were most often discovered by surveillance cystoscopy for bladder cancer $\left[{ }^{9]}\right.$.

In addition to the struggle of classifying lesions with identical histology, physicians have also investigated the designation of urinary tract pseudosarcomas as benign reactive change or potentially malignant neoplasms. While the histologic hallmarks of pseudosarcomas typically lack cellular atypia, active mitoses, and other signs of neoplastic change, their rapid and locally invasive growth patterns support their designation as neoplastic processes. It is crucial to understand the well-studied differences between pseudotumors and abdominal or pelvic sarcomas for this reason, as their gross appearance and locally invasive properties are strikingly similar. A recent study found that the key features of sarcomas separating the entity from pseudotumors were histologic features of nuclear atypia, mitotic figures, the presence of necrosis or myxoid degeneration, and predominantly neutrophilic inflammation [8]. It is important for physicians to understand the differences between pseudosarcomas and sarcomas, because the latter requires a larger and more expensive work-up as well as more advanced and aggressive therapy. Unlike sarcomas, metastasis has never been reported with pseudotumors despite their ability to have aggressively appearing, rapid local invasion. Thus, further imaging of chest, bone, and/or brain for potential metastasis are not warranted permitting the urologist has tissue evidence of a pseudotumor.

Sarcomas and pseudotumors of the urinary tract vary in their treatment regimen and prognoses. For both PSCN and PSFT, local excision is the gold standard treatment, whether by radical or partial cystectomy, prostatectomy, or transurethral resection (TUR). Transurethral resection has 
demonstrated success in the literature, however recurrence has only been reported with this type of treatment, inferring that it could be persistence from lack of negative margins rather than an actual recurrence. Death from disease is rare, with the most common result being obstructive urosepsis. Conversely, invasive sarcomas may also be treated with excision, but wider margins must typically be achieved. In addition, more aggressive treatment with chemotherapy may be indicated with these invasive sarcomas. Local recurrence and distant metastasis is common, and death has been reported in up to $50 \%$ of patients [9].

In conclusion, the rarity of pseudosarcomatous lesions in the urinary tract have led to sporadic reports of patterns regarding epidemiologic data, clinical presentation, and histologic morphology. In this case study, a 71-year-old gentleman without known history of instrumentation presented with recurrent painless, gross hematuria. Despite several studies' conclusions that postoperative spindle cell nodules tend to present later in life, this case emphasizes that these urinary tract pseudotumors may have protean presentations without precise epidemiologic trends. It is important for pathologists and urologists to consider medical and surgical history, the presence or absence of metastasis, and histologic findings to support an accurate diagnosis and direct appropriate therapy to avoid unnecessary radical measures.

\section{CONFLICT OF INTEREST}

The authors confirm that this article content has no conflict of interest

\section{ACKNOWLEDGEMENTS}

Declared none.

\section{REFERENCES}

[1] Roth JA. Reactive pseudosarcomatous response in urinary bladder. Urology 1980; 16: 635-7.

[2] Harik LR, Merino C, Coindre JM, et al. Pseudosarcomatous myofibroblastic proliferations of the bladder. A Clinicopathologic study of 42 cases. Am J Surg Pathol 2006; 30, 787-94.

[3] Cespedes RD, Lynch SC, Grider DJ. Pseudosarcomatous fibromyxoid tumor of the prostate. A case report with review of the literature. Urol Int 1996; 56: 249-51

[4] Horn LC, Reuter S, Biesold M. Inflammatory pseudotumor of the ureter and the urinary bladder. Pathol Res Pract 1997; 193, 607-12.

[5] Guillou L, Costa J. Postoperative pseudosarcomas of the genitourinary tract. A diagnostic trap. Presentation of 4 cases of which 2 were studied immunohistochemically and review of the literature. Ann Pathol 1989; 9, 340-5.

[6] Young RH, Scully RE. Pseudosarcomatous lesions of the urinary bladder, prostate gland, and urethra. A report of three cases and review of the literature. Arch Pathol Lab Med 1987; 111, 354-8.

[7] Proppe KH, Scully RE, Rosai J. Postoperative spindle cell nodules of the genitourinary tract resembling sarcoma: A report of 8 cases. Am J Surg Pathol 1984; 8: 101-8.

[8] Iczkowski KA, Shanks JH, Gadaleanu V, et al. Inflammatory pseudotumor and sarcoma of urinary bladder: differential diagnosis and outcome in thirty-eight spindle cell neoplasms. Mod Pathol 2001; 14(10): 1043-51.

[9] Spiess PE, Tuziak T, Tibbs RF, et al. Pseudosarcomatous and sarcomatous proliferations of the bladder. Human Pathol 2007; 38: 753-61. 